\title{
ANÁlISE ESTATÍSTICA PARA COMPARAÇÃO DAS CLASSES DE PERFIS DE SOLO DO ESTADO DO ACRE.
}

Lauro Magalhães Fráguas ${ }^{1}$

Ricardo Alexandrino Garcia²

\section{RESUMO}

O conhecimento dos solos e de sua capacidade de uso é de grande importância para o uso consciente e a preservação dos recursos a longo prazo. Para tanto, a elaboração, atualização e o controle da qualidade dos banco de dados referentes a solos é de suma importância. Nesse sentido este estudo objetivou a avaliação do banco de dados elaborado por AMARAL (2007). Utilizou-se Análise Discriminante para o agrupamento das classes de solo a nível de ordem. Das 516 amostras, somente 216 foram classificadas pela Análise Discriminante igual à existente no banco de dados. Com as amostras restantes, realizou-se uma ACP e os principais componentes encontrados estavam relacionados à fertilidade, mineralogia e acidez. Pelo teste de Turkey, determinou-se que houve confusão entre CAMBISSOLO e GLEISSOLO; e ESPODOSSOLO, ARGISSOLO, PLINTOSSOLO e LATOSSOLO.

\begin{abstract}
The knowledge of soils and its usability is of great importance for the wise use and conservation of resources in the long term. For this, the preparation, updating and quality control of database regarding land use is of paramount importance. In that sense, this study evaluates the database prepared by AMARAL (2007). Discriminant analysis was used for the grouping of soil types at the level of order. Of the 516 samples, only 216 were classified by discriminant analysis equal to that existing in the database. With the remaining samples, there was an ACP and the main components found were related to fertility, mineralogy and acidity. The test of Turkey, it was determined that there was confusion between INCEPTISOL and GLEISSOL, and SPODOSOLS, PODZSOIL, and PLINTHOSOL and LATOSOIL.
\end{abstract}

${ }^{1}$ CSR - Centro de Sensoriamento Remoto - UFMG

${ }^{2}$ Laboratório de estudos territoriais - LESTE/IGC/UFMG 


\section{1 - INTRODUÇÃO}

O conhecimento dos recursos naturais (solos, clima, vegetação e relevo) constitui parte do embasamento técnico-científico indispensável para a avaliação do potencial de uso das terras. Estas informações, combinadas com os contextos sociais, econômicos e culturais, levam à possibilidade de avaliação dos possíveis usos, das restrições e dos impactos ligados ao uso da terra.

Desse modo, é possível identificar áreas com maior ou menor aptidão para as mais diversas atividades, sejam agrícolas ou não, considerando aspectos sociais, econômicos e de responsabilidade no uso dos recursos naturais, visando benefícios coletivos. Desta maneira, a atividade de classificação dos solos possui uma importância enorme como ferramenta de tomada de decisão, e, para analisar fenômenos e planejar atividades de tal grandeza, torna-se indispensável um banco de dados consistente, georeferenciado e quantitativo (BENEDETTI et al., 2008).

Neste sentido, a partir de levantamentos de solo realizados pelo antigo Serviço Nacional de Levantamento e Conservação de Solos da Embrapa e órgãos antecessores, e pelo Projeto RADAM Brasil, foi desenvolvida uma base de dados de solos brasileiros (COOPER et al., 2005). Esta base, por falta de uma classificação unificada e atualizada dos perfis de solo, devido a mudanças em nomenclatura e critérios de distinção taxonômica no decorrer do tempo, permite apenas uma análise quantitativa e a classificação taxonômica não é tão precisa.

O estado do Acre é inserido neste contexto de preservação e uso racional dos recursos naturais e principalmente o solo de maneira peculiar. Além de estar localizada na região da Amazônia Ocidental, que vem sofrendo uma pressão de uso e uma ocupação crescente nas regiões de mata (AMARAL, 2007), os solos do Acre apresentam características próprias principalmente por serem de uma região de acumulo de sedimentos oriundos da Cordilheira dos Andes - dai a diversidade desses solos e características vérticas e de eutrofismo pouco comuns para a Amazônia. (ACRE, 2006).

O Zoneamento Ecológico-Econômico do Estado do Acre indica que as principais classes de solo presentes no estado em ordem decrescente de expressão territorial, são: ARGISSOLOS, CAMBISSOLOS, LUVISSOLOS, GLEISSOLOS, LATOSSOLOS, VERTISSOLOS, PLINTOSSOLOS e NEOSSOLOS; distribuídos conforme a Figura 1. 


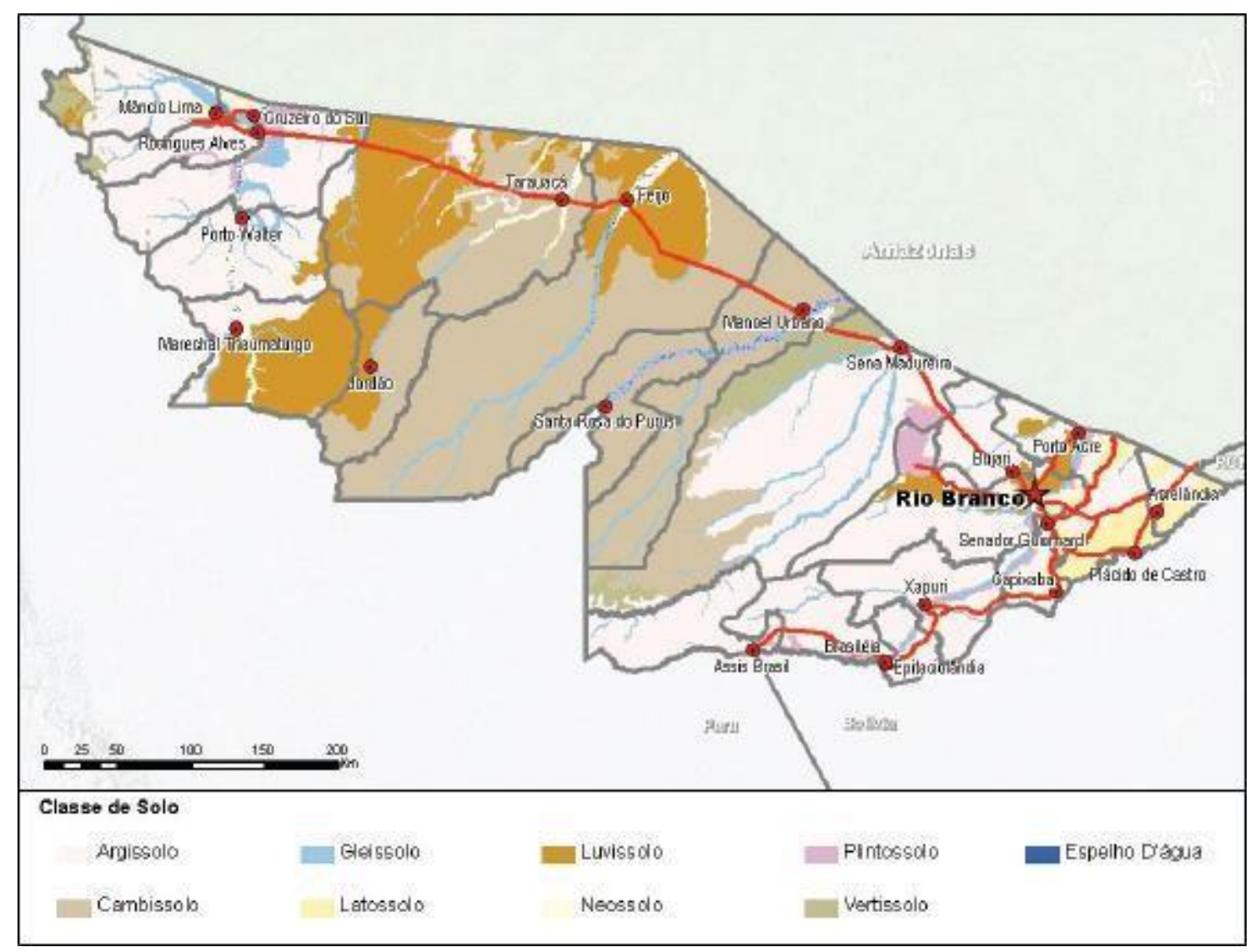

Figura 1: Distribuição simplificado dos solos do Acre, a nível de ordem. Fonte: Base de dados geográfico do ZEE/AC, 2006.

AMARAL (2007) propôs em seu estudo a construção de um banco de dados de solos para o estado do ACRE como instrumento de gestão ambiental. Este banco foi construído com dados de perfis de solos coletados pelo Projeto RADAM, pela EMBARAPA-AC, UFAC e por BARDALES (2005), totalizando 516 amostras de solo registradas. No entanto, existem as dificuldades de classificação já apresentadas para o banco de dados a nível nacional, além da peculiaridade da presença de características vérticas - argilas de alta reatividade - que fazem com que alguns perfis não sejam classificados totalmente nem na como a classe que até então fariam parte e, no entanto, também não possam ser considerados totalmente como Vertissolos.

Nesse contexto, o objetivo geral deste estudo é fazer uma análise estatística dos perfis do banco de dados relativo a solos elaborado por Amaral (2007) para o estado do Acre a fim de avaliar a classificação dos solos a nível de ordem. Os objetivos específicos do estudo são a avaliação da metodologia estatística para a análise, e a determinação de quais parâmetros são as principais fontes de confusão para na classificação dos perfis presentes no banco de dados. 


\section{2 - MATERIAIS E MÉTODOS}

Foi utilizado para a análise quantitativa deste estudo o banco de dados de solos elaborado por AMARAL (2007).O banco de dados possui 43 variáveis para cada perfil de solo. Estas variáveis vão desde as coordenadas geográficas do local de coleta do perfil, coordenadas físicas e químicas até a classificação dos solos segundo a Sociedade Brasileira de Ciência do Solo. Ao todo existem 516 perfis registrados, distribuídos nas classes ARGISSOLO, CAMBISSOLO, CHERNOSSOLO, ESPODOSSOLO, GLEISSOLO, LATOSSOLO, LUVISSOLO, NEOSSOLO,

LUVISSOLO, PLINTOSSOLO e VERTISSOLO. O software utilizado para os procedimentos estatísticos foi o SPSS Statics 17.0.

\begin{tabular}{|llll|}
\hline \multicolumn{3}{c|}{ Variáveis Utilizadas na Análise } \\
\hline Código do Perfil & Cor & $\mathrm{pH}$ em água & Sódio Trocável \\
Latitude & Teor de Areia Grossa & $\mathrm{pH}$ em Cloreto de Potássio & Alumínio Trocável \\
Longitude & Teor de Areia Fina & Carbono Total & Hidrogênio Trocável \\
Fonte & Teor de Silte & Matéria Orgânica & Acidez por Alumínio \\
Classe de Solo & Teor de Argila & Cálcio Trocável & Capacidade TrocaCatiônicaapH7 \\
Altitude & Silício Total & Magnésio Trocável & Soma de Bases \\
Drenagem & Óxidos de Alumínio Total & CalcioeMagnésioTrocáveis & Saturação de Bases \\
Profundidade do Solo & Óxidos de Ferro Total & Postássio Trocável & Saturação por Alumínio \\
\hline
\end{tabular}

Tabela 1: Parâmetros dos perfis utilizados na análise como variáveis.

\section{Pré-Tratamento da Variável Cor}

A cor do solo é uma variável categórica, formada por três valores numéricos, no formato “10YR 6/5”. As notações de matiz em número de sete, são representadas pelos símbolos 10R, 2,5YR, 5YR, 7,5YR, 10YR, 2,5Y e 5Y, que são formados pelas iniciais em inglês das cores que entram em sua composição (R - vermelho; Y - amarelo e YR de - vermelho-amarelo), precedidos de algarismos arábicos de 0 a 10, organizados a intervalos de 2,5 unidades. O segundo valor da notação indica maior ou menor participação do branco ou do preto (claridade ou escurecimento) em relação a uma escala neutra (acromática) e varia de 0 a 10. As notações de cromas indicam o grau de saturação pela cor espectral e variam de 0 a 8.

Para transformar o parâmetro cor em uma variável quantitativa, ela foi decomposta em 3 variáveis, representando cada uma das três escalas representadas no esquema de cor padrão no Sistema de Brasileiro de Classificação dos Solos. 


\section{Análise Discriminante}

Após o pré-tratamento na variável cor, o banco de dados passou por uma análise de discriminante, para a determinação a probabilidade de pertencimento dos perfis para cada uma das ordens de solo existentes no banco de dados.

A análise discriminante é uma "ferramenta" estatística utilizada para classificar um determinado elemento (E) num determinado grupo de variáveis; entre os diversos grupos existentes. Para tal é necessário que o elemento a ser classificado pertença realmente a um dos i grupos, e que sejam conhecidas as características dos elementos dos diversos grupos. Essas características são especificadas a partir de n variáveis aleatórias. No processo de classificação consideram-se os custos decorrentes de eventuais erros de classificação, bem como as probabilidades "a priori” de que o elemento pertença a cada um dos grupos (ANDERSON, 1984).

Assim, após a realização dos agrupamentos, considerou-se todas as amostras que possuíssem mais de 75\% de possibilidade de pertencer à classe na qual foi classificado como "tipos puros", quanto às outras amostras, foram selecionadas para uma análise de quais variáveis são responsáveis para a confusão entre os agrupamentos por ordens de solo.

\section{Análise de Componentes Principais (ACP)}

Logo em seguida, foi realizada uma análise de componentes principais, visando a redução das 32 variáveis analisadas em um número reduzido de componentes menos correlacionadas entre si. Desta forma, o processamento dos dados ocorre de maneira mais rápida e a análise dos resultados é menos custosa.

JOLLLIFFE (1986) define a ACP é um procedimento matemático que transforma uma série de variáveis correlacionadas em um número mais compacto de variáveis não correlacionadas chamadas componentes principais, relacionadas com as variáveis originais por uma transformação ortogonal. Esta transformação é definida de tal forma que o primeiro componente principal tem como uma alta variação possível, ou seja, representa o máximo da variabilidade dos dados quanto possível, e cada componente, por sua vez, tem a maior variância possível, sob a restrição de que seja ortogonal aos componentes anteriores. A ACP é sensível à escala relativa das variáveis originais. Devido a essa sensibilidade, os parâmetros de qualidade do solo podem ser todos inseridos na mesma análise, porque a diferença de unidade entre cada uma das variáveis utilizadas não interferirão nos resultados.

Neste trabalho, as 32 variáveis trabalhadas foram reduzidas no software a 6 componentes principais, no entanto as 3 primeiras componentes foram utilizadas na análise de diferença entre as 
médias para cada classe de solo, visto que somente elas já eram responsáveis por descrever o comportamento das amostras quase que na totalidade.

\section{Análise de Variância e Teste de Tukey}

Visando encontrar quais os principais fatores de confusão na classificação dos perfis, foi realizado o uma análise de variância (ANOVA) pelo teste, tendo como variáveis as 3 componentes principais mais representativas, e as ordens em que cada amostra possua maior probabilidade de pertencer, como fator de agrupamento das variáveis. Utilizou-se um intervalo de confiança de 95\%. Análise de variância é uma coleção de modelos estatísticos, e os seus procedimentos associados, no qual a observada variação em uma determinada variável é dividido em componentes atribuíveis a diferentes fontes de variação. Em sua forma mais simples, ANOVA fornece um teste estatístico da hipótese da média de vários grupos,e portanto, generaliza -teste t para mais de dois grupos.

Para as variáveis que foram rejeitados na hipótese de igualdade, aplicou-se o Teste de Tukey para saber quais as médias, entre as 10 classes de solos comparadas, diferem entre si ao nível de significância de $95 \%$.

\section{3 - RESULTADOS E DISCUSSÃO}

A Análise Discrimante foi realizada para todas os 516 perfis de solo .Apenas 246 perfis de solo foram classificados de acordo com a classificação já existente no banco de dados, de acordo com o padrão adotado na metodologia para a classificação. As outras 270 amostras foram classificadas incorretamente ou não atingiu a probabilidade mínima de $75 \%$ de pertencer ao seu agrupamento.

Todos os ARGISSOLOS que não atingiram o limite para ser classificados como tal na Análise Discriminante foram classificados como algum outro tipo de solo, em sua grande maioria foram classificados como LATOSSOLO e PLINTOSSOLO. Ressalta-se que nenhum ARGISSOLO foi classificado como tal com um percentual de pertencimento inferior ao limite arbitrado. 
No banco de dados existem 83 perfis de CAMBISSOLO, desses apenas 18 foram classificadas corretamente. Predominou a classificação dos CAMBISSOLOS como ARGISSOLOS ou CHERNOSSOLOS pelo modelo.

Os CHERNOSSOLOS constituem a classe com menos amostras. Das 8 amostras existentes, 7 foram classificadas corretamente e a restante foi identificada com CAMBISSOLO.

Os ESPODOSSOLOS também possuem 8 perfis registrados. Desses, 5 tiveram a mesma classificação pela Análise Discriminante e todas as outras 3 foram classificadas como LATOSSOLOS.

Existem no banco de dados 27 GLEISSOLOS, dos quais 16 não satisfizeram as condições do modelo estudado, sendo que 5 foram considerados VERTISSOLOS.

LATOSSOLOS possuem 33 perfis, sendo que 26 foram classificados como tal, não sendo possível identificar um padrão na classificação das outras 7 amostras.

O grupo da ordem dos LUVISSOLOS é constituído por 36 amostras, sendo que 21 não foram classificadas corretamente, sendo confundido com os CHERNOSSOLOS. Além dessas 21 amostras, outra foi classificada como CHERNOSSOLO com probabilidade de pertencimento superior a $75 \%$ e outras duas foram classificadas como VERTISSOLOS com mais de $75 \%$ de probabilidade. Este resultado pode ter ocorrido por se tratarem, as três classes, de solos com alto teor de argila de alta reatividade, diferenciando-se em outras propriedades e nas características do terreno onde são encontrados.

O agrupamento dos NEOSSOLOS possui 34 elementos, sendo que 12 não puderam ser identificados no modelo como tal. A classe com a qual houve mais confusão foi a dos VERTISSOLOS, com 5 elementos assim classificados. Os NEOSSOLOS são uma ordem de solo de difícil identificação por esta metodologia, visto que são muito heterogêneos e o único ponto em comum entre eles é a inexistência do horizonte $\mathrm{C}$, ou sua presença de forma incipiente.

Os PLINTOSSOLOS são 72 perfis, com apenas 17 classificados corretamente. Os outros 55 perfis são constituídos em sua grande maioria por GLEISSOLOS, ARGISSOLOS E CAMBISSOLOS, em ordem de representatividade. Os PLINTOSSOLOS possuem características físico-químicas muito próximas às das classes com as quais houve confusão, sendo a presença de concreções ferruginosas (plintitas) a característica determinante na classificação. 
VERTISSOLOS, os solos de característica mais peculiar no estado do Acre, por sua argila de alta reatividade, possui 26 amostras no banco de dados e, apenas 6 amostras foram classificadas erroneamente e não foi possível identificar em padrão nelas.

Com o total de 270 perfis de solos que não puderam ser identificados como pertencentes à classificação existente no banco de dados foi realizada a Análise de Componentes Principais. Segue abaixo as tabelas de outputs da ACP fornecidas pelo software.

Por meio da Tabela 3, pode-se inferir que as três primeiras dimensões da ACP são responsáveis por explicar quase a totalidade do comportamento das variáveis da análise.

A primeira componente é possui maior peso para Cálcio e Magnésio Trocáveis, Soma de Bases, Saturação por Bases, CTC a pH 7 e pH (ver Tabela 4). Essas variáveis são responsáveis e estão intimamente relacionadas à fertilidade do solo, portanto esta componente será nomeada como Fertilidade.

A componente 2 está fortemente relacionada à Óxidos Ferro, Alumínio e Silício Totais, Alumínio e Hidrogênio+Alumínio Trocáveis. Além disso há uma forte correlação negativa com Carbono e Matéria Orgânica. Portanto a componente 2 é relacionada à mineralogia dos solos, característica muito marcante em solos muito intemperizados tais como os LATOSSOLOS e ARGISSOLOS.

\begin{tabular}{|c|c|c|c|c|c|c|}
\hline & \multicolumn{6}{|c|}{ Dimension } \\
\hline & 1 & 2 & 3 & 4 & 5 & 6 \\
\hline Altitude & 288 & -208 &,- 095 &,- 011 & 646 &, 52 \\
\hline SoilDepth &,- 435 &,- 055 &,- 022 & ,163 &,- 492 &,- 10 \\
\hline Silt &, 547 &, 145 & ,151 & ,035 & 214 & -38 \\
\hline Clay & 029 & 820 & , 087 & 019 & 014 & ,162 \\
\hline TOTSIO2 & ,293 & ,650 &,- 168 & ,498 & 061 & ,01: \\
\hline TOTAL2O3 & ,022 & ,624 &,- 260 & ,624 & ,044 & 08 \\
\hline TOTFE2O3 & .111 &, 552 &,- 088 & ,625 &, 029 &,- 04 \\
\hline pH_H2O & ,595 &,- 276 &,- 493 & 170 &,- 008 &,- 12 \\
\hline pH_KCl &, 560 &,- 372 &,- 411 & 19d &,- 135 &, $04^{-}$ \\
\hline C & ,294 &,- 551 & ,644 & ,333 &,- 028 & 23 \\
\hline OM & ,29d &,- 560 & ,640 & ,334 &,- 014 & ,22 \\
\hline
\end{tabular}

Cadernos do Leste Artigos Cientificos 


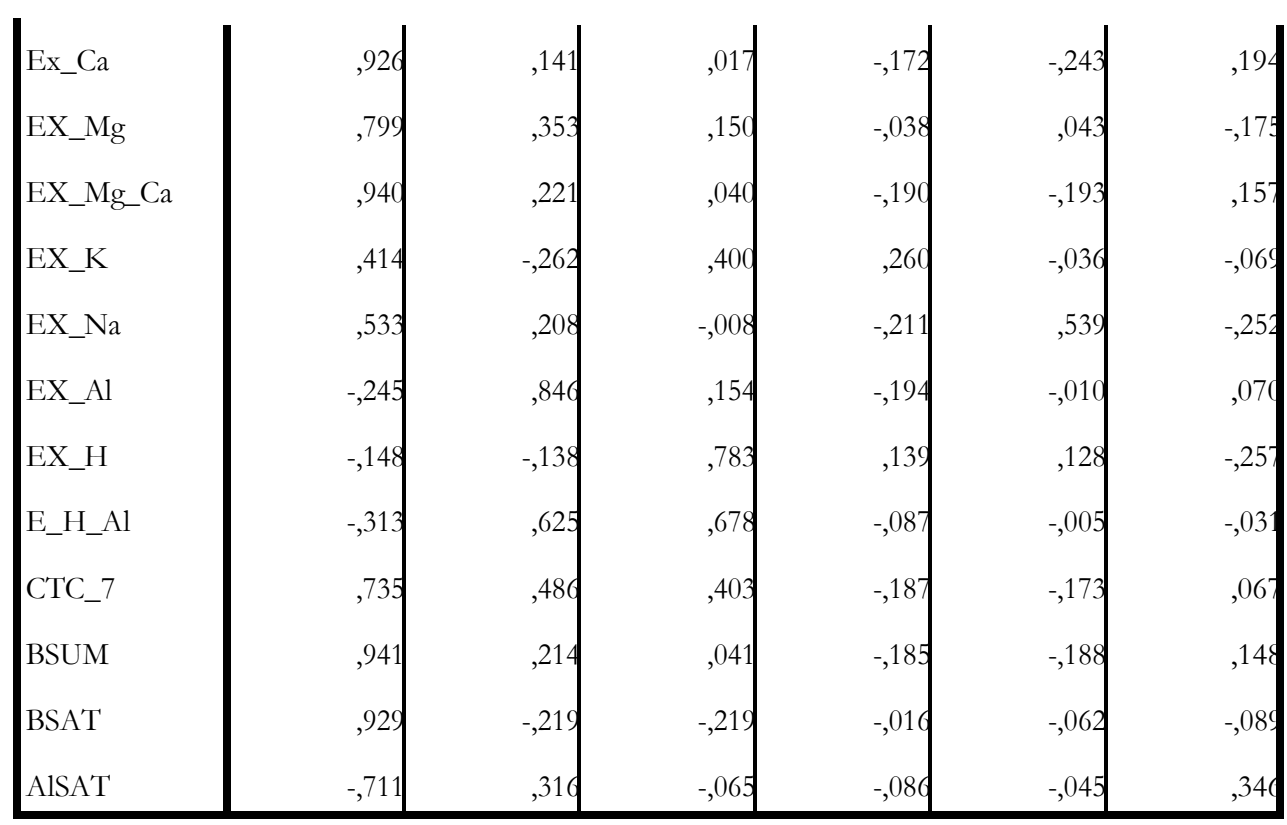

Variable Principal Normalization.

Tabela 4: Correlação das Componentes

A terceira e última componente utilizada no modelo, está fortemente correlacionada ao Hidrogênio e Hidrogênio+Alumínio Trocáveis, Carbono e Matéria Orgânica. Existe uma forte correlação negativa com o pH em Água e o pH em Cloreto de Potássio. Conclui-se que esta dimensão de informações está fortemente relacionada com a acidez do solo, fazendo destacar solos com $\mathrm{pH}$ baixo e alto teor de alumínio.

Com a ACP realizada e suas componentes devidamente selecionadas e analisadas, realizouse o Teste ANOVA e o Teste de Tukey Os outputs deste passo segue abaixo. Os códigos que variam de 2 a 11 significam, respectivamente: ARGISSOLO, CAMBISSOLO, CHERNOSSOLO, ESPODOSSOLO, GLEISSOLO, LATOSSOLO, LUVISSOLO, NEOSSOLO, PLINTOSSOLO E VERTISSOLO.

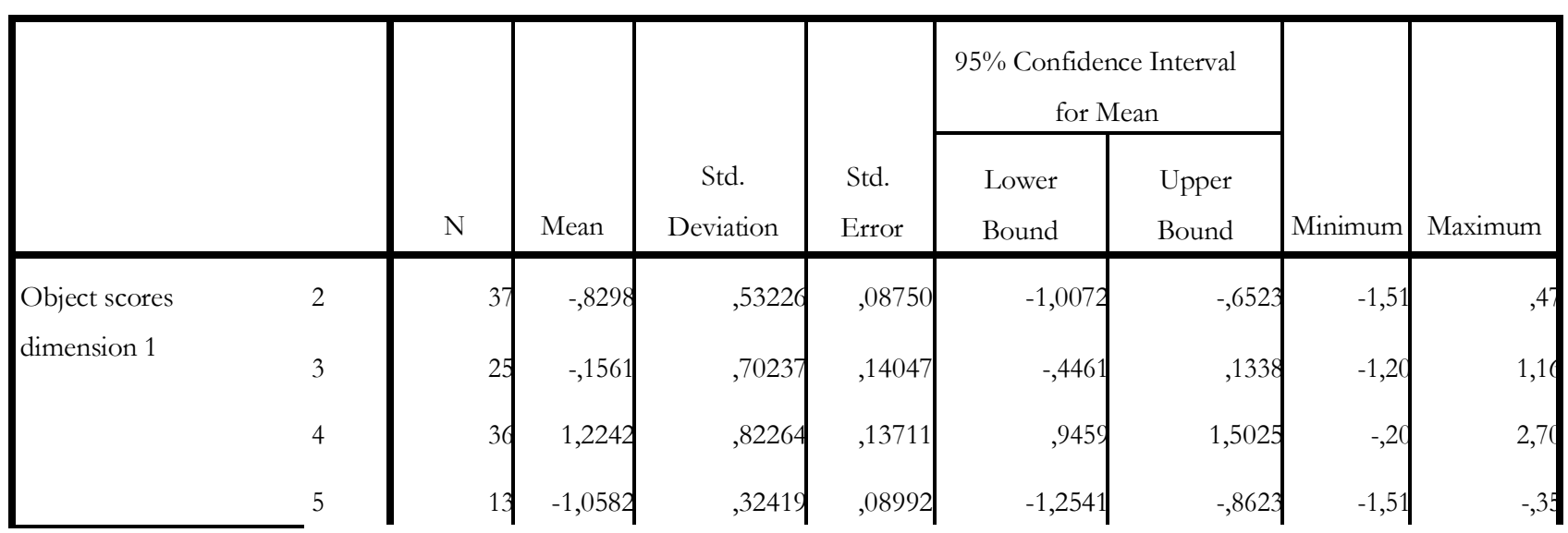




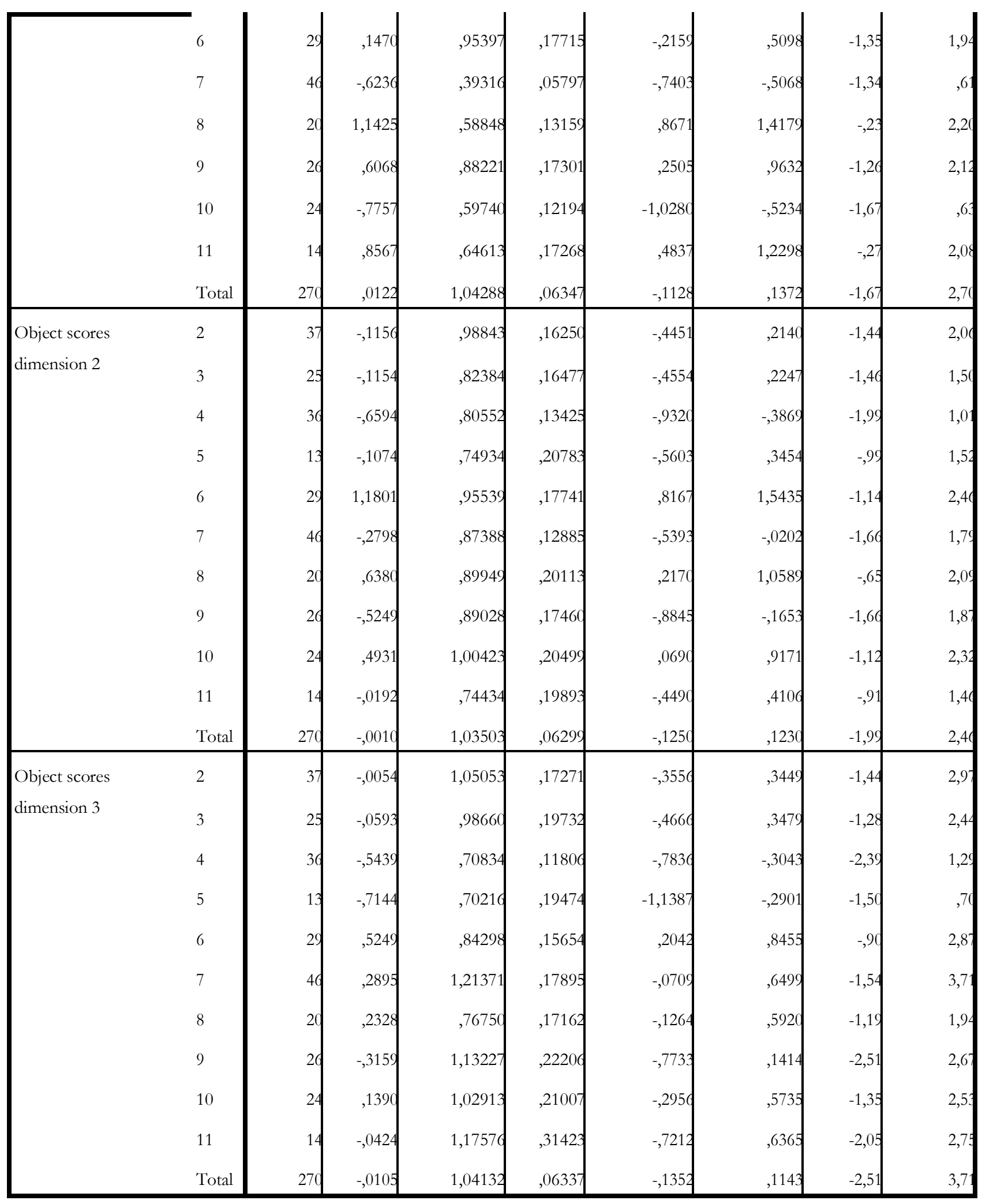

Tabela 5: Descrição das classes de solo em função das 3 componentes principais

ANOVA 


\begin{tabular}{|c|c|c|c|c|c|c|}
\hline & & Sum of Squares & df & Mean Square & $\mathrm{F}$ & Sig. \\
\hline Object scores dimension 1 & $\begin{array}{l}\text { Between Groups } \\
\text { Within Groups } \\
\text { Total }\end{array}$ & $\begin{array}{l}173,460 \\
119,090 \\
292,562\end{array}$ & 260 & $\begin{array}{r}19,274 \\
, 458\end{array}$ & 42,077 &, 000 \\
\hline Object scores dimension 2 & $\begin{array}{l}\text { Between Groups } \\
\text { Within Groups } \\
\text { Total }\end{array}$ & $\begin{array}{r}81,760 \\
206,410 \\
288,170\end{array}$ & 260 & $\begin{array}{r}9,084 \\
, 794\end{array}$ & 11,443 &, 000 \\
\hline Object scores dimension 3 & $\begin{array}{l}\text { Between Groups } \\
\text { Within Groups } \\
\text { Total }\end{array}$ & $\begin{array}{r}33,359 \\
258,328 \\
291,687\end{array}$ & 260 & $\begin{array}{r}3,707 \\
, 994\end{array}$ & 3,731 &, 000 \\
\hline
\end{tabular}

Tabela 6: Quadro de ANOVA para as três componentes principais

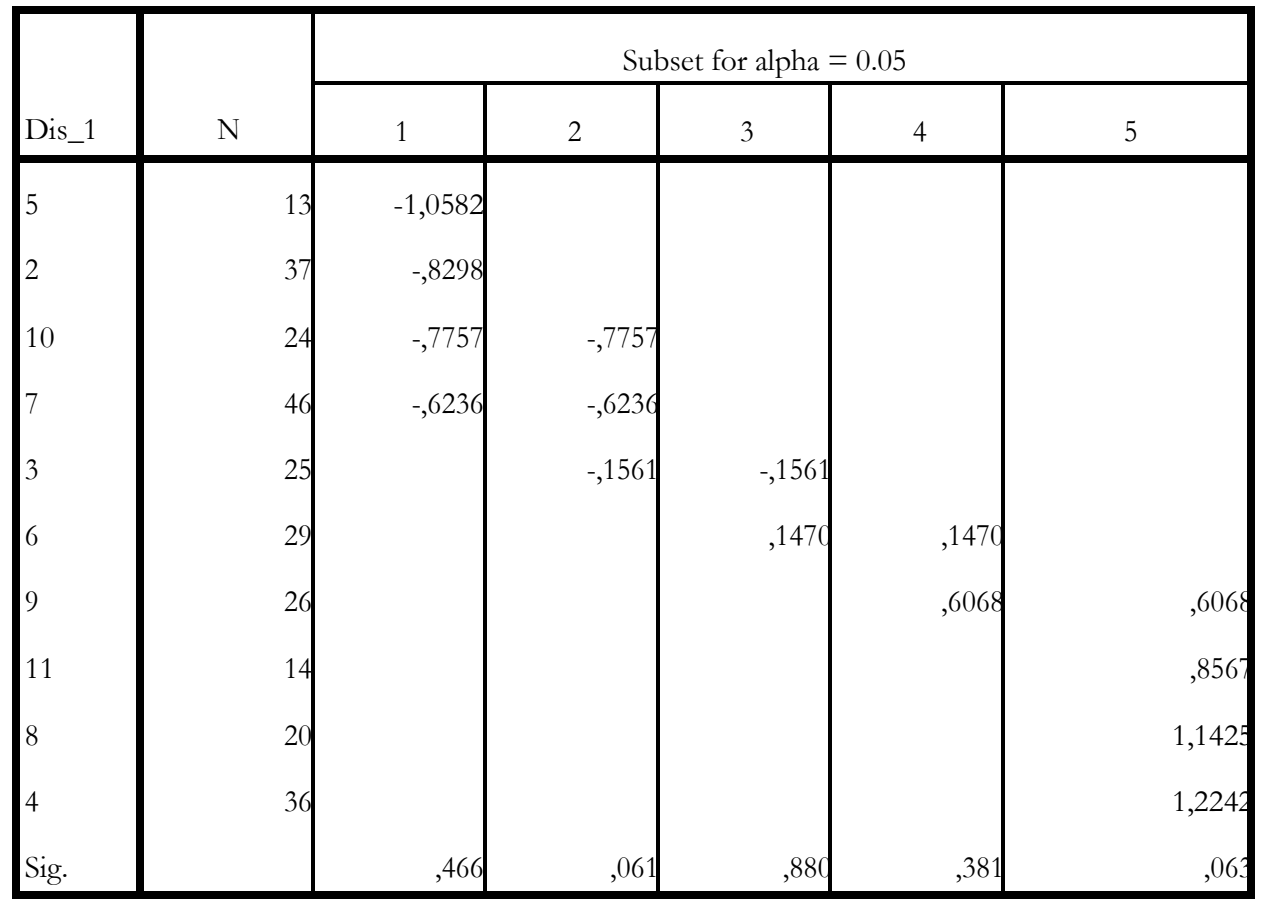

a. Uses Harmonic Mean Sample Size $=23,283$.

b. The group sizes are unequal. The harmonic mean of the group sizes is used. Type I error levels are not guaranteed.

Tabela 7: Agrupamento das classes de solo em função da diferença das médias dentro da componente 1. 


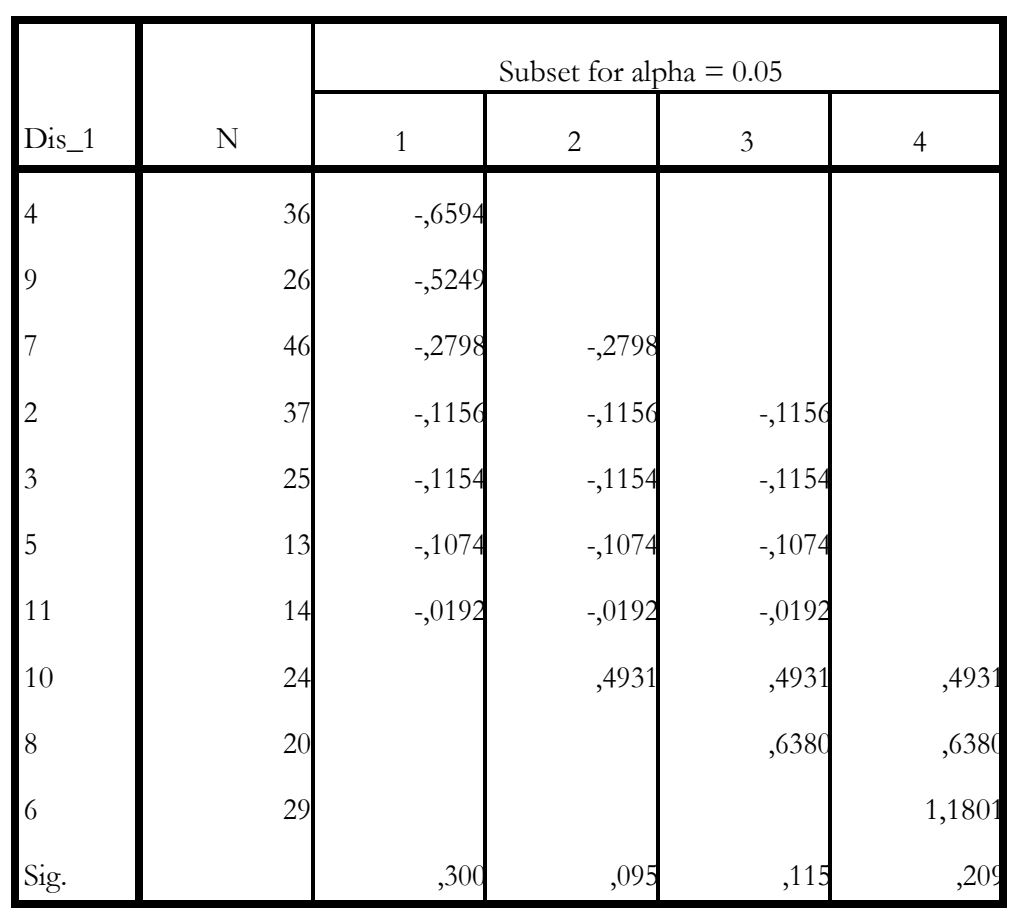

a. Uses Harmonic Mean Sample Size $=23,283$.

b. The group sizes are unequal. The harmonic mean of the group sizes is used. Type I error levels are not guaranteed.

Tabela 8: Agrupamento das classes de solo em função da diferença das médias dentro da componente 2.

\begin{tabular}{|c|c|c|c|c|}
\hline \multirow[b]{2}{*}{ Dis_1 } & \multirow[b]{2}{*}{$\mathrm{N}$} & \multicolumn{3}{|c|}{ Subset for alpha $=0.05$} \\
\hline & & 1 & 2 & 3 \\
\hline 5 & 13 &,- 7144 & & \\
\hline 4 & 36 &,- 5439 &,- 5439 & \\
\hline 9 & 26 & -3159 &,- 315 &,- 315 \\
\hline 3 & 25 & - -0593 &,- 0593 &,- 059 \\
\hline 11 & 14 & - -0424 &,- 0424 &,- 042 \\
\hline 2 & 37 & -0054 &,- 0054 &,- 0054 \\
\hline 10 & 24 & 1390 & 1390, & ,1390 \\
\hline 8 & 20 & & 2328 & 2328 \\
\hline 7 & 46 & & ,289月 & $289=$ \\
\hline 6 & 29 & & &, 5249 \\
\hline Sig. & & , 105 & , 12月 & 11 \\
\hline
\end{tabular}

Tabela 9: Agrupamento das classes de solo em função da diferença das médias dentro da componente 3.

O agrupamento apresentado na tabela 6 , apresenta uma confusão mais significativa entre CAMBISSOLO e GLEISSOLO. Outro agrupamento com significância superior a 0,5 foi o de 
número 1, que contém ESPODOSSOLO, ARGISSOLO, PLINTOSSOLO e LATOSSOLO.

Logicamente esses agrupamentos agregam classes de solo com características de ESPODOSSOLO, ARGISSOLO, PLINTOSSOLO e LATOSSOLO fertilidade semelhantes, sendo o CAMBISSOLO e o GLEISSOLO férteis geralmente e o outro grupo contendo solos de fertilidade baixa.

Já na componente 2, referente à mineralogia, somente o agrupamento 1 possui uma significância de 0,300 , todos os outros agrupamentos não possuem um valor de significância relevante. Este agrupamento é formado pela maior parte das classes de solos presentes no banco de dados: CHERNOSSOLO, NEOSSOLO, LATOSSOLO, ARGISSOLO, CAMBISSOLO, ESPODOSSOLO e VERTISSOLO. A presença de tantas classes dentro do mesmo agrupamento dificulta a análise desses tipos de solo, porém, é possível afirmar que eles são os solos com maiores teores de óxidos de ferro e alumínio em sua composição.

A terceira componente da ACP não possui nenhum agrupamento com maior significância para que seja explicada o padrão de aglomeração existente.

\section{4 - CONCLUSÃO}

O conjunto de métodos quantitativos apresentados neste trabalho foi capaz de identificar 246 amostras corretamente dentre os 516 existentes. Percebeu-se que a eficiência da Análise Discriminante foi diferente para cada classe de solos. Os ARGISSOLOS, grande maioria das amostras, com 217 elementos classificou apenas 86 de forma diferente à classificada anteriormente; enquanto dos 72 perfis de PLINTOSSOLOS, 17 foram classificados corretamente. Portanto o resultado da comparação entre a classificação realizada e a classificação modelada, não foi satisfatório, podendo ter seus resultados prejudicados pelos problemas na classificação já discutidos e por uma metodologia inadequada na confecção do modelo.

A ACP identificou que as três componentes principais mais importantes e principais fontes de confusão nas amostras não classificadas foram, em ordem de importância, fertilidade, mineralogia e acidez. O teste de Tukey conseguiu agrupar as classes de solo, em no máximo, 5 grupos, no caso da componente principal 1, sendo que apenas dois deles possuíam grande significância. 
Recomenda-se a realização deste mesmo procedimento, para cada uma das classes de solo isoladamente, a fim de comparar e aprimorar os resultados deste estudo.

\section{BIBLIOGRAFIA}

ACRE. Governo do Estado do Acre. Programa Estadual de Zoneamento Ecológico- Econômico do Estado do Acre. Zoneamento Ecológico- Econômico do Acre Fase II: documento Síntese - Escala 1:250.000. Rio Branco: SEMA, 2006. 356p.

AMARAL, E. F. do. Estratificação de ambientes para gestão ambiental e transferência de conhecimento, no Estado do Acre, Amazônia Ocidental. Viçosa, Universidade Federal de Viçosa, 2007. 185 p. (Dissertação de Doutorado).

Anderson, T.W., An Introduction to Multivariate Statistical Methods, 2a Ed., New York : John Wiley \& Sons, 1984.

BARDALES, N.G. Gênese, morfologia e classificação de solos do Baixo Vale do rio Iaco, Acre, Brasil. Universidade Federal de Viçosa. Viçosa, 2005. 132p. (Dissertação de Mestrado).

Benedetti M. M., Sparovek, G., Cooper, M., Representatividade e Potencial de Utilização de Utilização de Banco de Dados do Brasil, R. Bras. Ci. Solo, 32:2591-2600, 2008.

COOPER, M.; MENDES, L.M.S.; SILVA, W.L.C. \& SPAROVEK, G. A national soil profile database for Brazil available to international scientists. Soil Sci. Soc. Am. J., 69:649-652, 2005.

Jolliffe, I. T., Principal Component Analysis. 1a Ed. Springer-Verlag pp. 487, 1986 Virgillito, S. B., Estatística Aplicada, 3a Ed. Edicon, PP. 590, 2008. 